\title{
NEIGHBOR SUM DISTINGUISHING TOTAL CHOOSABILITY OF IC-PLANAR GRAPHS
}

\author{
Wen-Yao Song, Lian-Ying Miao \\ School of Mathematics \\ China University of Mining and Technology \\ Xuzhou 221116, P.R. China \\ e-mail: songwenyao@cumt.edu.cn \\ miaolianying@cumt.edu.cn \\ AND \\ YUAN-YUAN DUAN \\ School of Mathematics and Statistics \\ Zaozhuang University \\ Zaozhuang 277160, P.R. China \\ e-mail: duanyy0827@sina.com
}

\begin{abstract}
Two distinct crossings are independent if the end-vertices of the crossed pair of edges are mutually different. If a graph $G$ has a drawing in the plane such that every two crossings are independent, then we call $G$ a plane graph with independent crossings or IC-planar graph for short. A proper total- $k$ coloring of a graph $G$ is a mapping $c: V(G) \cup E(G) \rightarrow\{1,2, \ldots, k\}$ such that any two adjacent elements in $V(G) \cup E(G)$ receive different colors. Let $\sum_{c}(v)$ denote the sum of the color of a vertex $v$ and the colors of all incident edges of $v$. A total- $k$-neighbor sum distinguishing-coloring of $G$ is a total- $k$ coloring of $G$ such that for each edge $u v \in E(G), \sum_{c}(u) \neq \sum_{c}(v)$. The least number $k$ needed for such a coloring of $G$ is the neighbor sum distinguishing total chromatic number, denoted by $\chi_{\Sigma}^{\prime \prime}(G)$. In this paper, it is proved that if $G$ is an IC-planar graph with maximum degree $\Delta(G)$, then $\operatorname{ch}_{\Sigma}^{\prime \prime}(G) \leq$ $\max \{\Delta(G)+3,17\}$, where $c h_{\Sigma}^{\prime \prime}(G)$ is the neighbor sum distinguishing total choosability of $G$.
\end{abstract}

Keywords: neighbor sum distinguishing total choosability, maximum degree, IC-planar graph, Combinatorial Nullstellensatz.

2010 Mathematics Subject Classification: 05C15. 


\section{REFERENCES}

[1] M.O. Albertson, Chromatic number, independence ratio, and crossing number, Ars Math. Contemp. 1 (2008) 1-6.

[2] N. Alon, Combinatorial Nullstellensatz, Combin. Probab. Comput. 8 (1999) 7-29. doi:10.1017/S0963548398003411

[3] J.A. Bondy and U.S.R. Murty, Graph Theory with Applications (North-Holland, New York-Amsterdam-Oxford, 1982).

[4] L. Ding, G. Wang and G. Yan, Neighbor sum distinguishing total colorings via the Combinatorial Nullstellensatz, Sci. China Math. 57 (2014) 1875-1882. doi:10.1007/s11425-014-4796-0

[5] L. Ding, G. Wang, J. Wu and J. Yu, Neighbor sum (set) distinguishing total choosability via the Combinatorial Nullstellensatz, Graphs Combin. 33 (2017) 885-900. doi:10.1007/s00373-017-1806-3

[6] A. Dong and G. Wang, Neighbor sum distinguishing total colorings of graphs with bounded maximum average degree, Acta Math. Sin. (Engl. Ser.) 30 (2014) 703-709. doi:10.1007/s10114-014-2454-7

[7] D. Král and L. Stacho, Coloring plane graphs with independent crossings, J. Graph Theory 64 (2010) 184-205. doi:10.1002/jgt.20448

[8] H. Li, B. Liu and G. Wang, Neighbor sum distinguishing total colorings of $K_{4}$-minor free graphs, Front. Math. China 8 (2013) 1351-1366. doi:10.1007/s11464-013-0322-x

[9] H. Li, L. Ding, B. Liu and G. Wang, Neighbor sum distinguishing total colorings of planar graphs, J. Comb. Optim. 30 (2015) 675-688. doi:10.1007/s10878-013-9660-6

[10] S. Loeb, J. Przybyło and Y. Tang, Asymptotically optimal neighbor sum distinguishing total colorings of graphs, Discrete Math. 340 (2017) 58-62. doi:10.1016/j.disc.2016.08.012

[11] M. Pilśniak and M. Woźniak, On the total-neighbor-distinguishing index by sums, Graphs Combin. 31 (2015) 771-782. doi:10.1007/s00373-013-1399-4

[12] C. Qu, G. Wang, J. Wu and X. Yu, On the neighbor sum distinguishing total coloring of planar graphs, Theoret. Comput. Sci. 609 (2016) 162-170. doi:10.1016/j.tcs.2015.09.017

[13] C. Qu, G. Wang, G. Yan and X. Yu, Neighbor sum distinguishing total choosability of planar graphs, J. Comb. Optim. 32 (2016) 906-916. doi:10.1007/s10878-015-9911-9

[14] D.E. Scheim, The number of edge 3-colorings of a planar cubic graph as a permanent, Discrete Math. 8 (1974) 377-382. doi:10.1016/0012-365X(74)90157-5 
[15] J. Wang, J. Cai and Q. Ma, Neighbor sum distinguishing total choosability of planar graphs without 4-cycles, Discrete Appl. Math. 206 (2016) 215-219.

doi:10.1016/j.dam.2016.02.003

[16] J. Yao and H. Kong, Neighbor sum distinguishing total choosability of graphs with larger maximum average degree, Ars Combin. 125 (2016) 347-360.

[17] X. Zhang and J. Wu, On edge colorings of 1-planar graphs, Inform. Process. Lett. 111 (2011) 124-128.

doi:10.1016/j.ipl.2010.11.001

[18] J. Yao, X. Yu, G. Wang and C. Xu, Neighbor sum (set) distinguishing total choosability of d-degenerate graphs, Graphs Combin. 32 (2016) 1611-1620. doi:10.1007/s00373-015-1646-y

[19] X. Cheng, D. Huang, G. Wang and J. Wu, Neighbor sum distinguishing total colorings of planar graphs with maximum degree $\Delta$, Discrete Appl. Math. 190-191 (2015) 34-41.

doi:10.1016/j.dam.2015.03.013

[20] Y. Lu, M.M. Han and R. Luo, Neighbor sum distinguishing total coloring and list neighbor sum distinguishing total coloring, Discrete Appl. Math. 237 (2018) 109-115.

doi:10.1016/j.dam.2017.12.001

Received 30 June 2017

Revised 21 March 2018

Accepted 21 March 2018 